\title{
Graphene Is Thin, but Not Infinitely So
}

\section{Atomically thin graphene is considered a prototypical 2D material, but high-pressure experiments now reveal the 3D nature of its mechanical properties.}

\section{by John Proctor*}

W e live in a 3D world, yet $2 \mathrm{D}$ materials are one of the hottest topics in physics right now. Graphene (Fig. 1) is perhaps the most famous example. Quite simply, the material "looks" $2 \mathrm{D}$ because it is only one atom thick. But does it act 2D? In terms of electronic properties, the answer is yes, as graphene has an electronic band structure that is different from its 3D counterpart, graphite. Similarly, graphene's thermal expansion exhibits telltale 2D characteristics. But what about graphene's mechanical properties? Yiwei Sun of Queen Mary University of London and colleagues have addressed this question by subjecting this prototypical 2D material to a traditional 3D experiment [1]. The team compressed flakes of graphene to a pressure of $12 \mathrm{GPa}(120,000$ times greater than atmospheric pressure) and showed that the resulting energy shift of graphene's vibrations (phonons) is consistent with a 3D material rather than a $2 \mathrm{D}$ one. The finding may have implications for applications of graphene and other

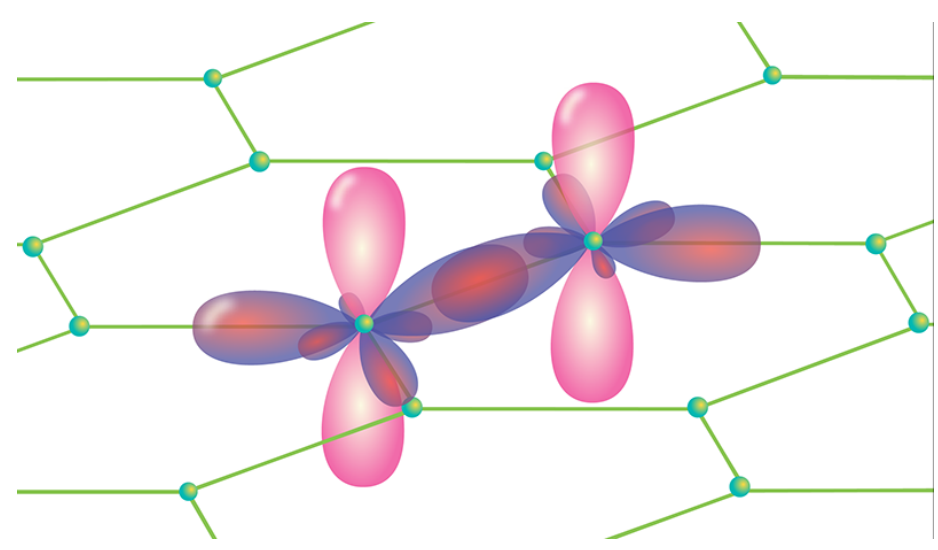

Figure 1: Graphene is only one carbon atom thick and looks 2D. But it has electronic orbitals that extend perpendicularly to the atomic plane, such as the $2 p_{z}$ orbitals (light pink). These orbitals resist compression in the direction perpendicular to graphene's lattice, a characteristic of 3D materials. (APS/Carin Cain)

\footnotetext{
* School of Computing, Science \& Engineering, University of Salford, Salford, United Kingdom
}

2D materials as mechanical sensors and structural reinforcements.

The first thing to clarify is that no completely flat, freestanding material would ever be stable. The reason is that phonons perpendicular to the 2D plane will, colloquially speaking, always shake the material apart at any temperature above absolute zero [2-4]. The root of this effect is how phonons behave in a $2 \mathrm{D}$ material: the phonon density of states is linear with wave vector $k$, resulting in a lot of phonons at low energy. In comparison, the density of phonon states in 3D materials falls off much faster at low energy because it varies as $k^{2}$. Theorists knew the disruptive effect of phonons long before 2D materials came into fashion [2], so discovering a genuinely stable 2D material would have raised some serious theoretical problems.

Researchers have been able to stabilize graphene and related families of materials by supporting them on a substrate-though the materials still have ripples in their shape. However, the use of a substrate means that measurements of graphene's properties have to account for the effect of the material support. About a decade ago, my colleagues and I turned this problem into an opportunity by using measurements on a graphene sample supported by a substrate and at high pressure to infer properties of graphene under known strain [5]. In our experiment, the graphene adhered strongly to the substrate, so any compression of the substrate translated into a known strain on the graphene. But because of the substrate, such an experiment can't directly determine the stress on graphene, which is the parameter used in models of mechanical properties.

In their work, Sun et al. go one better than the previous study. They do away with the supporting substrate by suspending flakes of monolayer graphene in a viscous liquid [1]. The liquid prevents the flakes from crumpling and/or bonding together to form graphite for long enough to perform an experiment. With this approach, an applied pressure on the liquid will translate directly into a known stress on the graphene.

Sun et al. squeezed the graphene-containing liquid between two anvil-shaped diamonds-a setup known as a diamond anvil cell (DAC). Using optical Raman spectroscopy, they made a detailed and careful study of the vibrations in the graphene sheet at high pressure. Their analysis demonstrates that-as far as mechanical properties are concerned-we cannot treat graphene as a fully 2D material. 
Specifically, they find that the vibrations shift to higher values with pressure. The same qualitative behavior is seen in both 2D and 3D materials, but to accurately model and explain the observed shifts in graphene, Sun et al. had to treat it as a 3D material with an out-of-plane elastic constant-meaning graphene can become thinner under compression. Graphene, it seems, is more like cardboard than a sheet of paper.

High-pressure experiments like these are easy to describe, but they are notoriously difficult to perform. Fiddly handiwork is required to align the DAC and sample with micrometer-precision. Because of these demands, such experiments also have a high failure rate. Sun and colleagues' ability to study graphene under a known high stress-a first-is therefore a major achievement.

The team's results will also have concrete implications. If graphene were infinitely thin, one would only need to know its in-plane elastic constant to work out how it would behave under any kind of stress. The fact that graphene is 3D means the out-of-plane direction matters as well. From a microscopic perspective, this conclusion makes sense: although graphene is one atom thick, each atom has electronic orbitals (the $2 p_{z}$ orbitals) that extend some distance above and below the graphene sheet and resist compression (Fig. 1). Therefore, it is meaningful to describe elastic properties in relation to this axis as well as the in-plane axes.

We can expect to see this research used in the development of strain sensors based on graphene. It may also affect how Raman spectroscopy is used as a diagnostic tool for new types of graphene composites that serve to reinforce other materials. Here, the spectroscopy helps determine the extent to which stress or strain is transferred from the host material to the graphene reinforcement. Knowing graphene's 3D characteristics will help researchers optimize this reinforcing behavior.

This research is published in Physical Review Letters.

\section{REFERENCES}

[1] Y. W. Sun, W. Liu, I. Hernandez, J. Gonzalez, F. Rodriguez, D. J. Dunstan, and C. J. Humphreys, "3D strain in 2D materials: To what extent is monolayer graphene graphite?" Phys. Rev. Lett. 123, 135501 (2019).

[2] A. K. Geim and K. S. Novoselov, "The rise of graphene," Nat. Mater. 6, 183 (2007).

[3] A. H. Castro Neto, F. Guinea, N. M. R. Peres, K. S. Novoselov, and A. K. Geim, "The electronic properties of graphene," Rev. Mod. Phys. 81, 109 (2009).

[4] J. E. Proctor, D. A. Melendrez Armada, and A. Vijayaraghavan, An Introduction to Graphene and Carbon Nanotubes (CRC Press, Boca Raton, 2017).

[5] J. E. Proctor, E. Gregoryanz, K. S. Novoselov, M. Lotya, J. N. Coleman, and M. P. Halsall, "High-pressure Raman spectroscopy of graphene," Phys. Rev. B 80, 073408 (2009).

10.1103/Physics. 12.104 